\title{
Sex specific effects of alterations in macronutrient composition on fat accumulation, lipid-synthesis, -transport and -storage
}

\author{
Kirstin Andersen ${ }^{1}$, L. Kavermann ${ }^{1}$, A. Horngacher ${ }^{1}$, R. Z. Tom ${ }^{2}$, S. Sachs ${ }^{2}$, S. Hofmann ${ }^{2}$, M. Bidlingmaier ${ }^{1}$ \\ 1 Endocrine Research Unit, Medizinische Klinik und Poliklinik IV, Klinikum der Universität München, Munich, Germany
}

2 Helmholtz Zentrum München, German Research Centre for Environmental Health Institute of Diabetes and Regeneration Research, Neuherberg, Germany Contact: Martin.Bidlingmaier@med.Imu.de / KAnderse@med.Imu.de

Introduction

Recent data suggest that not only caloric density but also macronutrient composition of diets can influence metabolism, body composition and the development of metabolic disorders. A limitation of previous studies was that effects were studied predominantly in males. To better understand potential differences in the response of males and females to manipulations of dietary macronutrients, our studies were undertaken in both sexes.

\section{Methods}

As a dietary model, we used regular chow diet $(\mathrm{CH})$, low carbohydrate-high fat diets of normal (LCHF-1) and low (LCHF-2) protein content (ketogenic) as well as a high carbohydrate-high fat diet (HCHF). Purified diets (\% of metabolizable energy, fat/protein/carbohydrate: Chow (CH, 16.7/19.0/64.3), protein matched LCHF-1 (78.7/19.1/2.2), ketogenic LCHF-2 (92.8/5.5/1.7) and HCHF (61.9/18.7/19.4)) were pairfed isoenergetically for 4 weeks to male and female Wistar rats (12 weeks at start, $n=7 /$ group). At study end ( 6 hours fasting, dark phase), blood samples and organs were collected.

\section{Results}

Compared to $\mathrm{CH}$, all isoenergetically fed diets high in fat led to reduced body weight (BW) gain. This effect was seen in both sexes and was independent of carbohydrate content. However, despite lower BW, animals fed LCHF-1 and LCHF-2 but not HCFC exhibited a significant $\left({ }^{*} \mathrm{p}<0.05 ;{ }^{* * *} \mathrm{p}<0.001\right)$ increase in visceral fat $(\% ; \mathrm{g} / \mathrm{g} \mathrm{BW})$ in both sexes (male CH: $0.93 \pm 0.07$, LCHF-1: $1.22 \pm 0.07^{* * *}$, LCHF-2: 1.26 $\pm 0.07^{* * *}, \mathrm{HCHF}$ $1.08 \pm 0.06$, female CH: $0.65 \pm 0.05$, LCHF-1: $0.93 \pm 0.08$, LCHF-2: $1.03 \pm 0.09^{* * *}$, HCHF: $0.64 \pm 0.05)$. Notably, subcutaneous fat mass increased significantly only in males. Higher serum leptin was seen in all animals fed HF-diets (male CH: 2837 \pm 374 , LCHF-1: 4094 3366 , LCHF-2: 4646 $\pm 617^{*}$, HCHF: 4599 $\pm 411^{*}$, female CH: 904 \pm 193 , LCHF-1: 2433 $\pm 434^{*}$, LCHF-2: $2355 \pm 446^{*}$, HCHF: $2157 \pm 311$ ). In subcutaneous white adipose tissue (s.c. WAT) a pronounced upregulation in adipokines like adiponectin (AdipoQ) and leptin (LEP) mRNA expression was seen only in males. Whereas on protein leve Leptin was increased in both genders when fed a high fat diet. As expected higher leptin levels were detectable in all diets in male rats. Furthermore, surrogate markers of lipid synthesis (FASN), transport (LPL) and storage (ATGL) in subcutaneous WAT were also increased only in male animals on high fat diets.

Conclusion

These data strongly suggest that macronutrients affect lipid metabolism and storage in a sex specific manner. Compared to females, males have increased lipid synthesis, transport and storage, higher triglycerides and WAT weight, and increased hepatic lipid content. Interestingly, "browning" of WAT as mirrored by Cidea expression seems to be increased in animals fed LC diets, with particularly pronounced effects in males.
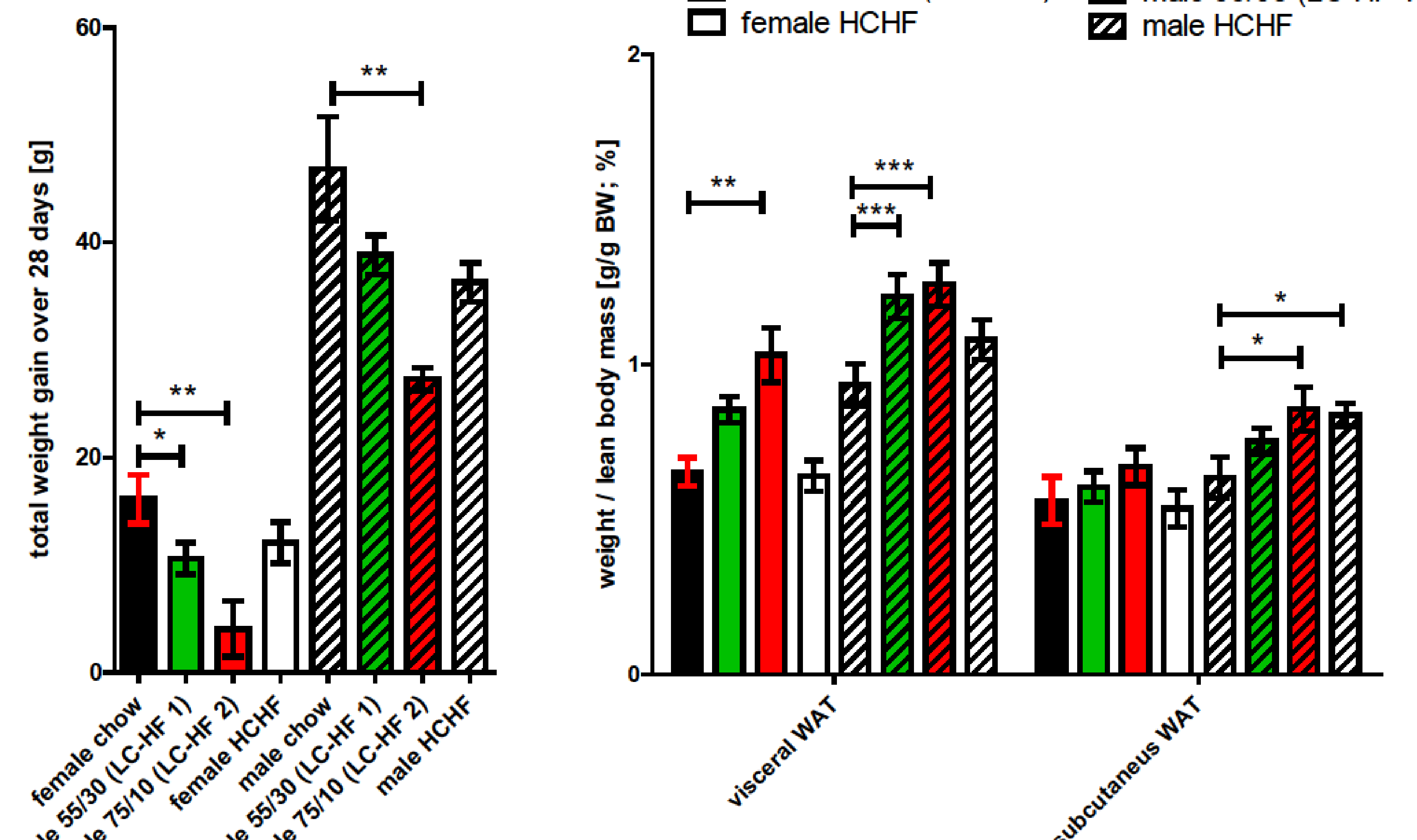
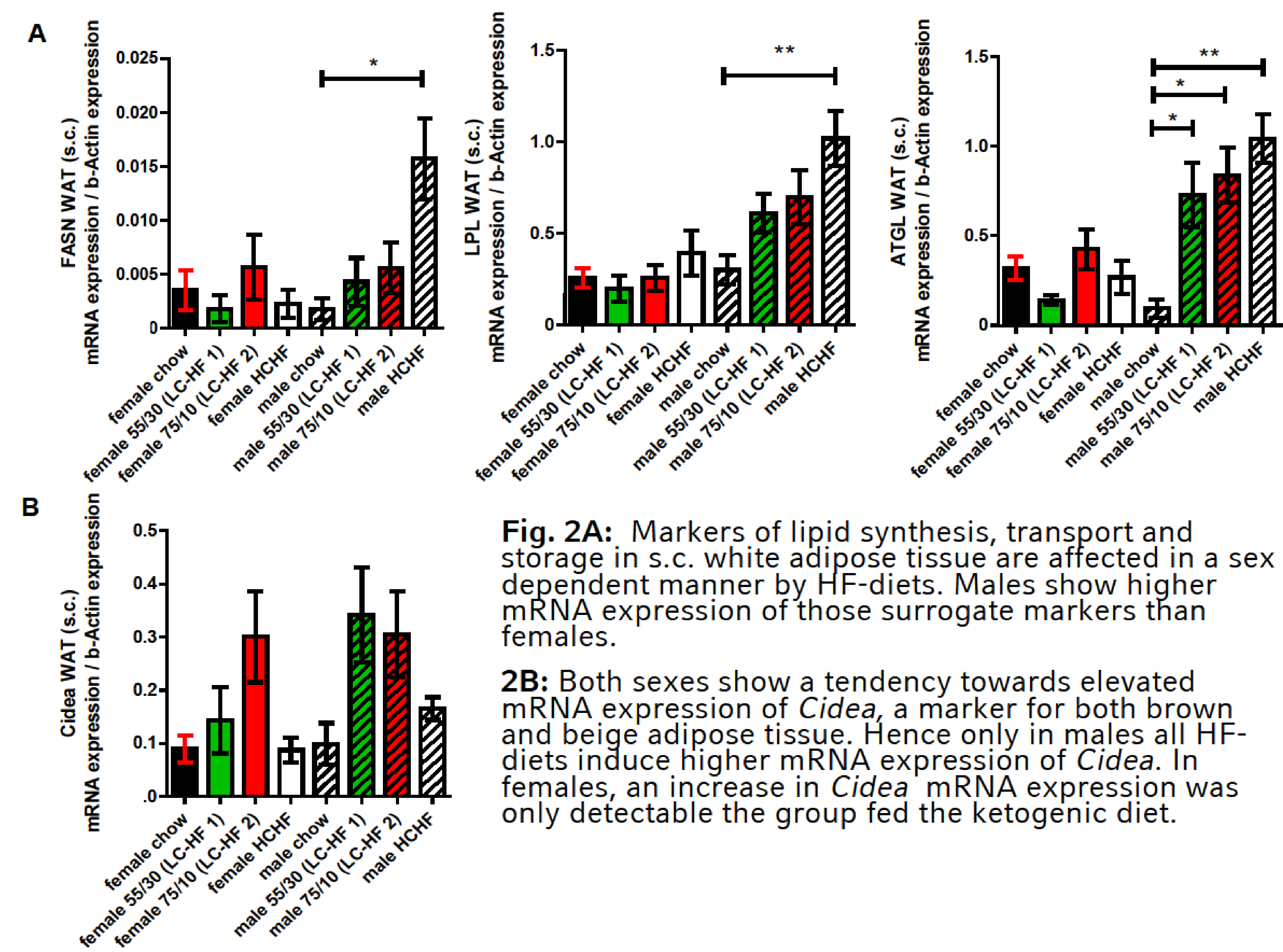

Fig. 2A: Markers of lipid synthesis, transport and in a sex mRNA expression of those surrogate markers than females.

2B: Both sexes show a tendency towards elevated and beige adipose tissue. Hence only in males all HFdiets induce higher mRNA expression of Cidea. In females, an increase in Cidea mRNA expression was only detectable the group fed the ketogenic diet.
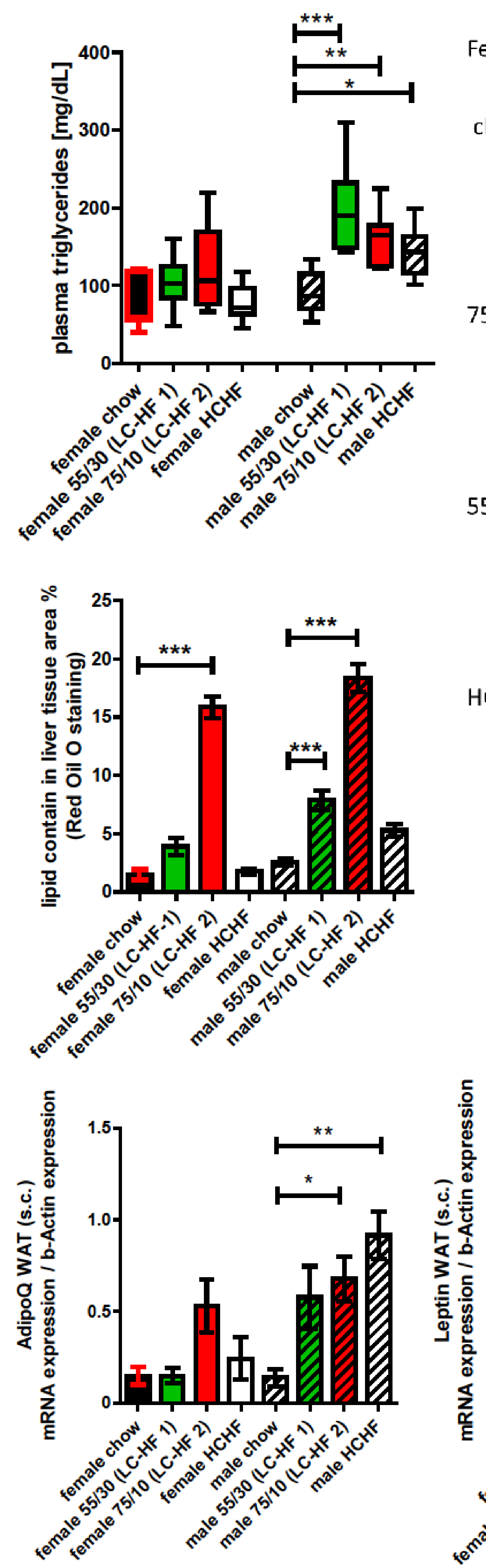

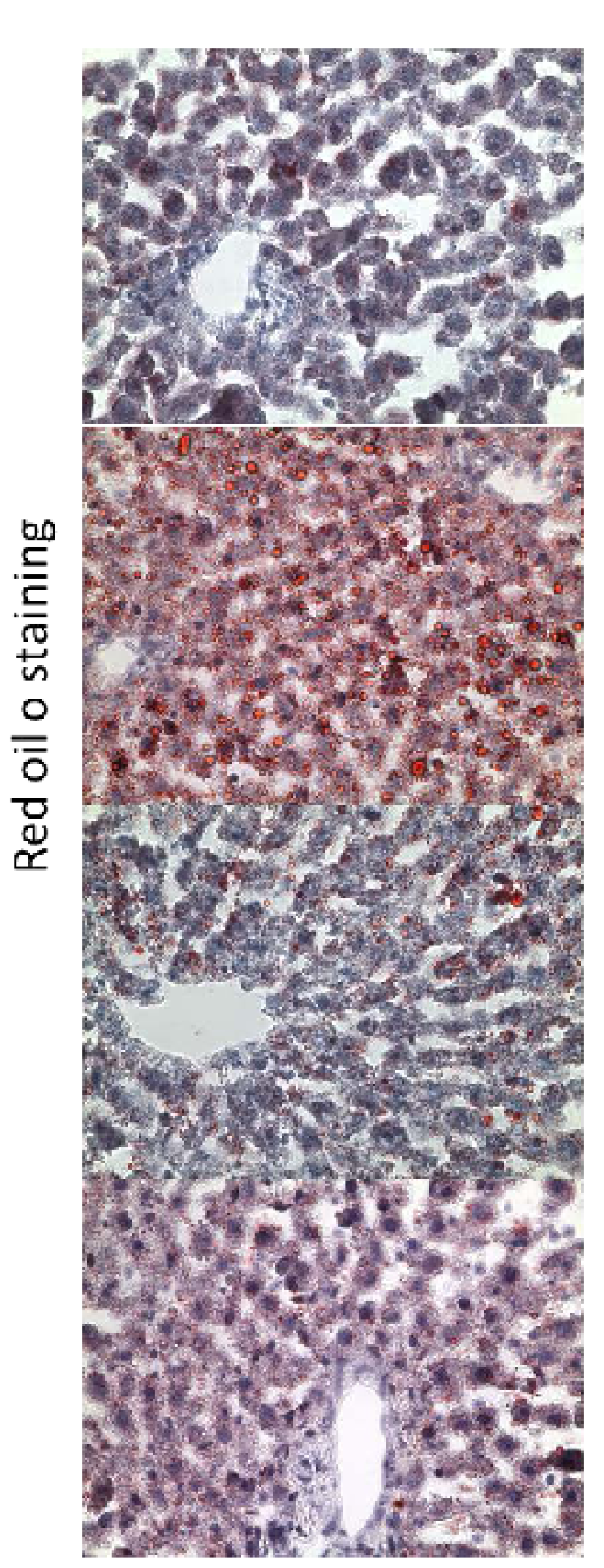

$75 / 10$

Fig. 3: Liver lipid content is similar in male and female

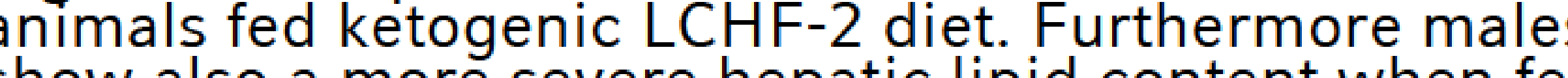
LCHF-1 or HCHF diets. In line with the histological findings serum triglyceride levels are elevated more pronounced in males

Fig. 1: Animals on all HF-diets show reduced weight gain in both sexes. However, relative change in total weight gain over 28 days did not show differences between sexes. Changes in WAT (white adipose tissue) in HF-diet fed animals compared to chow fed animals. In both types of WAT a more pronounced increase was seen in male animals.
Fig. 4: $m R N A$ levels for adipokines were upregulated in all male animals fed HF-diets in contrast females show only a tendency of elevation when fed ketogenic HF-diet. On protein level an increase of serum leptin in both genders was detectable.

Supported by a grant from the

Else Kröner-Fresenius-Stiftung (2014_A108) 